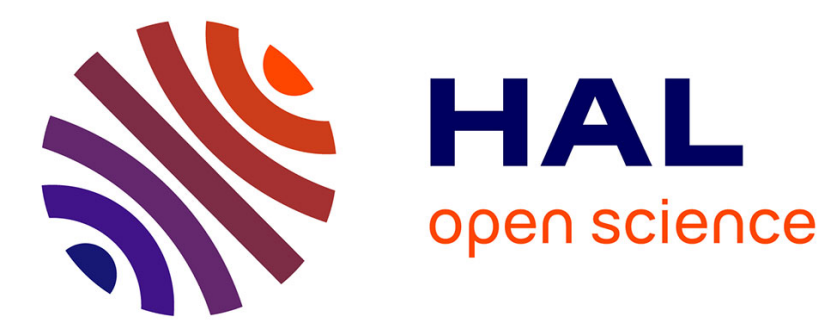

\title{
Etude sur les non-usagers d'Internet: analyse de la perception des TIC et du rapport aux médias
}

\author{
Feirouz Boudokhane-Lima
}

\section{To cite this version:}

Feirouz Boudokhane-Lima. Etude sur les non-usagers d'Internet: analyse de la perception des TIC et du rapport aux médias. Les Enjeux de l'information et de la communication, 2012. sic_01228016

\section{HAL Id: sic_01228016 \\ https://archivesic.ccsd.cnrs.fr/sic_01228016}

Submitted on 12 Nov 2015

HAL is a multi-disciplinary open access archive for the deposit and dissemination of scientific research documents, whether they are published or not. The documents may come from teaching and research institutions in France or abroad, or from public or private research centers.
L'archive ouverte pluridisciplinaire HAL, est destinée au dépôt et à la diffusion de documents scientifiques de niveau recherche, publiés ou non, émanant des établissements d'enseignement et de recherche français ou étrangers, des laboratoires publics ou privés. 


\section{Etude sur les non-usagers d'Internet : analyse de la perception des TIC et du rapport aux médias}

Feirouz Boudokhane Lima

\section{Introduction}

L'individu se sert généralement comme cadre de référence de ce qu'il a connu et expérimenté pour appréhender les choses qu'il ignore. Les innovateurs d'un nouveau produit sont généralement des personnes qui ont une expérience significative avec des catégories d'objets similaires (cf. Balloffet, Boulaire, 1999). Ceci pourrait être dû au fait que les usagers, familiers d'un type de dispositif, ont des schémas cognitifs plus développés à l'égard de ce genre d'outil et ont, ainsi, moins de problèmes à comprendre et à accepter une autre innovation du même registre (Ibid.). Dans ce contexte, on peut penser que les rapports, perceptions et expériences négatives d'un individu avec certaines TIC peuvent l'amener à rejeter d'autres catégories de technologies considérées comme similaires.

Ce travail de terrain est une étude sur les non-usagers d'Internet; il s'articule autour de la question de leur rapport aux autres TIC (ordinateur, téléphone portable, appareils numériques, ...). Nous ne cherchons pas à dresser un portrait-type du non-usager. Nous nous interrogeons sur l'influence de leur perception de ces outils quant au refus du réseau. Quelles représentations les non-usagers d'Internet ont-ils des autres TIC ? Quelles expériences ont-ils de ces technologies?

Nous nous intéressons enfin aux usages que font les enquêtés des médias dits «traditionnels » (livre, radio, télévision). Quelle comparaison font-ils entre l'Internet et ces médias ?

L'article est structuré ainsi en deux parties. La première est centrée sur le rapport des nonusagers d'Internet aux autres TIC (ordinateur, téléphone portable, appareils numériques, ...), et la deuxième partie est centrée sur le rapport des enquêtés aux médias «traditionnels ».

Pour essayer d'apporter des éléments de réponse à notre problématique nous avons opté pour une étude qualitative basée sur des entretiens semi-directifs. Notre échantillon est constitué de 25 non-usagers. Ces personnes ont entre 33 et 77 ans, parmi eux 14 sont de sexe masculin et 11 de sexe féminin. Ils habitent la Communauté Urbaine de Bordeaux (la CUB) et appartiennent à différentes catégories socioprofessionnelles: bibliothécaire, ouvriers, employés, artisans, enseignants, retraités, sans-emploi.

Nous avons pris contact avec les enquêtés en recourant à la méthode dite de proche en proche. Celle-ci « consiste à demander à un premier interviewé potentiel de désigner d'autres interviewés possibles et ainsi de faire la chaîne » (Blanchet, Gotman, 1992, p. 58). Certaines personnes de notre entourage ont permis d'amorcer cette chaîne qui s'est ensuite prolongée par la désignation d'autres réfractaires par les premiers interviewés.

Il était difficile de rencontrer des non-usagers (dont le non-usage est volontaire). Ces individus ne forment pas un groupe et ils n'appartiennent pas à des réseaux identifiés. Ainsi, et pour augmenter les chances de voir acceptées nos demandes d'entretien, le mode d'accès aux interviewés qui paraissait le plus propice au recueil de données était la méthode « de proche en proche ».

Les informations recueillies ont fait l'objet d'une analyse de contenu. Il était question plus spécifiquement d'une analyse thématique. Nous avons effectué une analyse individuelle de chaque entretien, ce qui nous a permis de nous imprégner des différents propos et de dégager 
l'éventail de thèmes. Cette première analyse a été suivie d'une autre transversale et thématique de l'ensemble des entretiens. Les réponses retranscrites ont été classées par thèmes, après avoir été synthétisées selon un procédé de découpage conseillé par Laurence Bardin (1977). L'analyse de contenu «vise la simplification des contenus : elle a pour fonction un effet d'intelligibilité et comporte une part d'interprétation » (Blanchet, op.cit., p.92). Parmi ses variantes, l'analyse thématique semblait être la plus à même pour exploiter au mieux les réponses et les propos des enquêtés dans le cadre de ce travail. Cette analyse nous a permis de repérer les thèmes communs et transversaux à l'ensemble des entretiens recueillis. De façon générale et pour mener à bien cette méthode d'analyse les opérations suivantes ont été effectuées :

- Nous avons lu plusieurs fois et de manière approfondie et attentive chaque entretien afin de faire l'analyse individuelle. L'analyse a porté ensuite sur le corpus dans sa totalité en repérant les thèmes dans chaque entretien.

- Nous avons sélectionné dans l'ensemble des entretiens tout ce qui est utile pour notre sujet à savoir le rapport aux TIC et aux médias chez les non-usagers d'Internet. Nous avons en effet trié notre matériau en fonction des thèmes retenus pour l'analyse. Il s'agit de découper les entretiens en utilisant des ciseaux et de mettre ensemble tout ce qui concerne un même thème. - Au cours de notre analyse thématique, nous avons utilisé des méthodes qui relèvent de l'analyse de discours : étude du vocabulaire usité, relevé des termes ayant un sens plutôt positif ou négatif. Nous avons aussi fait appel à des méthodes quantitatives : comptage de récurrences, du nombre de fois où une idée se répétait dans plusieurs entretiens. Nous avons également fait un "travail de va-et-vient" entre ce que nous avons pu découvrir dans les entretiens, nos notes de lecture et notre problématique.

Ce travail prend appui sur une étude théorique que nous avons réalisée en 2006 et intitulée «Comprendre le non-usage technique : réflexions théoriques ». En se basant sur des études appartenant aux champs de la sociologie (notamment la sociologie de la diffusion et celle de la consommation), de la psychosociologie, des sciences de gestion et des sciences de l'information et de la communication, nous avons identifié dans ce travail théorique des éléments explicatifs du non-usage, qui sont principalement : les facteurs liés aux caractéristiques de la technique et celles liées aux caractéristiques de l'individu.

La présente étude, qui est un travail de terrain, s'inscrit également dans la problématique du non-usage. Nous avons ainsi voulu vérifier les éléments identifiés lors de notre étude théorique sur le non-usage. En effet, nous postulons que les caractéristiques perçues des TIC et d'Internet ont une influence sur le rapport des enquêtés à ces technologies.

Il s'agit notamment des caractéristiques suivantes : l'inconvénient perçu (S. Ram, E. Rogers) ; les risques perçus: risque fonctionnel ( $\mathrm{S}$. Ram), risque social (S. Ram, S. Latouche, D. Wolton, P. Breton) ; l'incompatibilité perçu (E. Rogers) ; les faibles possibilités d'essai et de transfert (S. Ram, U. Zander et B. Kogut).

Nous postulons également que les caractéristiques propres aux enquêtés déterminent fortement leur rapport à Internet et aux autres TIC. Il s'agit des caractéristiques suivantes : l'auto-efficacité perçue (R. Wood, A. Bandura) ; les représentations, les expériences, le besoin et les attitudes face à l'innovation (P. Mallein, M. Rokeach); les caractéristiques sociodémographiques (S. Ram). 


\section{Le rapport des réfractaires d'Internet aux autres TIC}

Une étude par entretien implique une écoute fine, de la patience, de l'objectivité et un grand intérêt envers le sujet de recherche. Les entretiens ont été réalisés en tenant compte des principes énoncés par Moliner et al. (2002) : suivre le sujet au lieu de le diriger, l'inciter à approfondir sa pensée, favoriser la progression de l'entrevue. C'est par ce type de démarche que nous avons essayé d'interroger les réfractaires à Internet sur leur rapport aux autres TIC (ordinateur, téléphone portable, appareils numériques, ...).

Ce qui ressort des discours recueillis, c'est la diversité des situations et des représentations. Les expériences ainsi que les perceptions et attitudes des enquêtés envers les autres technologies ont une influence non négligeable sur leur refus de l'Internet.

Certains non-usagers ne cachent pas leur peur d'être envahis par les TIC et insistent sur leur volonté d'être indépendants de ces outils. Leurs réticences vis-à-vis des TIC s'expliquent par le refus et la peur d'être soumis au diktat de la technique, d'où les déclarations suivantes : «ça me fait peur, la technique prend trop le pas sur l'homme»; «avec les appareils numériques c'est à la personne de s'adapter aux exigences et aux caprices de ces techniques». Jean-Éric (48 ans) nous a raconté, par exemple, qu'il utilisait très peu de moyens de communication par souci d'avoir « un minimum de liberté » (1). Il refuse ainsi, comme d'autres enquêtés, d'être enchaîné par «les fils invisibles de la communication » (cf. Wolton, 2000). De même, lorsqu'on a demandé à Richard (38 ans) de nous parler de ses rapports aux TIC, il nous a d'emblée dit qu'il trouvait ces techniques très envahissantes : «On peut dire qu'on perd énormément de personnalité. On est envahi par ces appareils ». Les TIC sont perçues par nos interlocuteurs comme «omniprésentes», «accablantes ». Certains trouvent qu'on est en train de perdre le contrôle de ces engins. Ils considèrent les nouvelles technologies comme des moyens qui « dépossèdent l'homme de sa liberté à agir et l'asservit à son propre mode de fonctionnement ». Nos interlocuteurs veulent échapper à la puissance aliénatrice de la technologie, qui prive l'homme d'une dimension essentielle de la liberté humaine, la possibilité même de choisir (cf. Alain Gras, 2003). Pour eux, les TIC dicteraient une temporalité et une spatialité spécifiques, auxquelles les individus seraient obligés de s'adapter. Ces outils enchaîneraient leurs utilisateurs et leurs feraient oublier d'autres types de temporalité comme le temps familial et privé.

Plusieurs interrogés refusent d'être équipés de certaines technologies comme par exemple : l'appareil photo numérique, le lecteur DVD et Blu-Ray, le GPS, l'ordinateur, le téléphone portable, le baladeur MP3, MP4, etc. Et même ceux dont le foyer dispose de certains appareils, nous ont avoué que ce sont leurs enfants ou leur conjoint qui s'en servent.

Le non-usage de nos interlocuteurs s'explique parfois par le manque d'intérêt pour les TIC, la non-utilité et le non besoin perçus. Le sociologue Philippe Mallein postule que l'utilité précède l'usage, ainsi la perception de la non-utilité d'un objet technique peut déterminer l'absence de son usage. Il est fréquent d'entendre dire chez les non-usagers rencontrés : "Je ne m'intéresse pas aux nouvelles technologies »; «Ce n'est pas ma tasse de thé »; "Je n'ai aucun de ces appareils numériques, ça ne m'intéresse pas!»; «Je n'ai pas besoin de ces technologies ».

Les enquêtés ont donc évoqué un désintérêt total pour les TIC. Certains disent que ces dispositifs représentent « un nouveau système commercial », et d'autres pensent qu'ils ne sont 
pas concernés par les technologies. Ces dernières seraient «destinés aux riches », ou encore aux « hommes d'affaire et professionnels du commerce ». D'autres réfractaires disent que les nouvelles technologies n'ont «aucune utilité », ils déclarent ainsi que leur intérêt ne se dirige pas vers le fait d'avoir «le dernier gadget inventé ».

Certains enquêtés trouvent que les TIC «accentuent la rapidité des modes de vie». Ils pensent qu'il faut être «jeune» pour pouvoir utiliser ces outils et suivre l'évolution technologique dans ce domaine. Le facteur de l'âge semble expliquer la réticence de certains enquêtés vis-à-vis des TIC. Les propos suivants sont révélateurs de cette perception : "Pour les gens de mon âge, ça dépasse un peu ce qu'on imagine. On a à peine le temps de s'adapter à quelque chose que déjà ils ont inventé une autre. A chaque fois j'entends parler d'un nouveau truc, c'est fou! »(Colette, 71 ans), «Ce n'est plus de mon âge, toutes ces nouvelles technologies sont destinées aux jeunes »(Germaine, 77 ans).

La majorité des non-usagers qui ont plus que 50 ans ont évoqué la question de l'incompatibilité des technologies numériques avec leur âge. Les personnes âgées se sentent souvent exclues face aux nouvelles technologies. Philippe Breton dit dans ce contexte : «Le culte de l'Internet est un culte jeune, de jeunes et pour les jeunes. (...). On peut se demander, à lire certains articles et certaines déclarations, quelle place "le nouveau monde" laisse aux vieux. [Il existe] un véritable discours d'exclusion des personnes âgées dans le domaine des nouvelles technologies de l'information » (p.87-89).

Plusieurs actions ont été menées par les pouvoirs publics pour réduire la fracture numérique et notamment le fossé générationnel. Plusieurs rapports préconisent la familiarisation des personnes âgées aux outils du numérique grâce à un accompagnement personnalisé et des logiciels ou des matériels adaptés leur permettant par exemple de correspondre plus aisément avec leurs proches ou de bénéficier d'un suivi médical à distance (2). En dépit des moyens proposés le fossé générationnel en France reste important. D'après un rapport publié par le centre d'analyse stratégique le 4 avril 2011 et intitulé «Le fossé numérique en France », seuls $16,9 \%$ des plus de 75 ans et $40 \%$ des 55-64 ans disposent à leur domicile d'un ordinateur contre $91 \%$ pour les $15-24$ ans.

Il n'est pas envisageable d'imposer une technologie à une génération pour que celle-ci l'utilise. Le non-usage des nouvelles technologies chez les personnes âgées n'est pas toujours un choix délibéré. Leur non-usage est souvent lié à des dimensions cognitives consécutives au vieillissement (3), au manque de connaissance technique ou encore à l'absence de besoin : «Toutes ces technologies sont compliquées pour moi, il y a trop de touches sur un portable ou sur un ordinateur, il est difficile à mon âge de mémoriser tout cela! Et puis il y a toujours une nouvelle chose, je ne peux pas suivre toutes ces innovations et je n'ai pas à le faire, je n'ai absolument pas besoin de ces outils» (Colette, 71 ans).

Plusieurs de nos interlocuteurs regrettent certains engins et expriment leur attachement à des outils auxquels ils se sont habitués : par exemple le magnétoscope par rapport au DVD, l'appareil photo analogique par rapport au numérique. Certaines personnes âgées disent même continuer à écouter les 33 tours ou encore les cassettes.

Les réfractaires à Internet n'hésitent pas à dénoncer les pratiques que permettraient certaines nouvelles technologies. Plusieurs évoquent par exemple le cas des appareils photos numériques qui permettraient de truquer les photos et de les modifier sans limites. Nos interlocuteurs font souvent une comparaison entre les appareils analogiques et les numériques. L'ancien est toujours présenté comme quelque chose de « solide », «fiable », « authentique » et le nouveau comme «fragile» et «incertain». Ainsi pour l'appareil photo numérique, on 
trouve les propos suivants : "Avant, prendre des photos c'était un art. Maintenant on fait n'importe quoi! On peut tricher, truquer, enlever ou ajouter quelqu'un sur une photo. Je trouve ça terrifiant! »(Daniel, 52 ans) ; "Je n'ai aucun rapport avec les nouvelles technologies, ça ne m'intéresse pas! Je n'ai pas Internet, je n'ai pas d'appareils numériques. Je ne suis pas un adepte du numérique. Je préfère faire des vraies photos à l'ancienne » (Jacques, 63 ans).

Par ailleurs, certains enquêtés soulignent dans leurs discours le fait qu'ils préfèrent le contact direct à toutes les nouvelles techniques de communication. Le téléphone fixe reste le moyen le plus utilisé après la communication orale. Cette dernière serait considérée comme la base de tout rapport entre les humains. Certains n'admettent pas que la communication puisse se réaliser autrement que dans la proximité. Le téléphone, Internet ou autres pourraient servir à échanger un message ponctuel, mais ne permettraient pas de communiquer dans le but de partager, de se comprendre et de créer des liens sociaux.

En effet, le risque social perçu, relevé par Ram dans $A$ model of innovation resistance, explique les réticences de certains enquêtés vis-à-vis des TIC. Les propos suivants reflètent cette perception : «les nouvelles technologies détruisent les relations humaines »; "Tout ça est antinomique avec le relationnel humain et classique »; «du moment où on passe par l'intermédiaire d'une machine on ne peut pas appeler ça communication!».

Les non-usagers dénoncent la facilité de l'acte de communication. Ce dernier nécessiterait, selon nos interlocuteurs, une certaine préparation et une sorte de réflexion. Or, la facilité avec laquelle on pourrait rentrer en contact avec des inconnus via les nouvelles technologies abolirait tous ces critères. La communication deviendrait ainsi vidée de tout sens, de toute profondeur.

Les réticences de certains non-usagers vis-à-vis des TIC s'expliquent également par l'incompatibilité perçue de l'objet, ce point a déjà été relevé par Rogers dans son fameux Diffusion of innovations. Les propos suivants sont révélateurs de la perception de l'incompatibilité des TIC avec les préférences et les habitudes de certains enquêtés : «Je préfère aller vers les gens, voir la personne. Internet et toutes ces technologies modernes ne correspondent pas à mon mode de vie » (Daniel) ; "Chaque génération a ses trucs, je ne fais pas partie de cette génération moi. Nous on n'est pas habitué à tout ça! Je vois ma petite fille comment elle utilise Internet, le portable, l'ordinateur, tout ça elle l'a dans le sang! » (Giselle, 64 ans).

L'incompatibilité renvoie au degré avec lequel une innovation est perçue comme nonconforme aux valeurs existantes, aux habitudes, aux expériences passées et aux besoins du récepteur (cf. Rogers, 2003). Un dispositif technique risque d'être rejeté s'il exige un haut degré de changement et de réajustement de la part des usagers potentiels. Selon Rogers, certains individus peuvent être attachés à leurs habitudes, à leurs façons de faire, si bien qu'il n'est pas simple de leurs faire accepter les changements. La perception de la part d'un individu de l'incompatibilité d'un objet technique avec son style de vie peut justifier son nonusage.

Par ailleurs, les enquêtés font énormément allusion aux contraintes et aux aléas de la technique. Ils évoquent par exemple les risques fonctionnels des TIC, notamment celles liées à l'informatique: virus, panne, incertitude de performance, obsolescence, complexité, etc. Certains disent ainsi ne pas vouloir perdre leur temps, ou avoir «des problèmes en plus » à cause de ces techniques qui « compliquent la vie». 
Parmi les TIC les plus contestées, on trouve notamment le téléphone portable : «Je déteste le portable, on est toujours alerté par la sonnerie pour un oui ou un non » (Jean-Éric) ; «Je ne suis pas du tout portable, je ne supporte pas l'idée qu'on puisse me sonner n'importe où et à n'importe quel moment » (Nathalie, 45 ans).

Certaines personnes âgées interrogées, font un rapprochement entre le téléphone portable et Internet et évoquent la complexité de ces technologies pour eux. Dans ce contexte, Germaine nous a décrit l'expérience décevante qu'elle a eu avec le portable et nous a avoué que cela ne l'encourage pas à utiliser Internet parce qu'elle trouve très compliquées ces technologies auxquelles elle n'est pas habituée. La complexité perçue de la technique, déjà relevée par Rogers (2003) et Ram (1987), semble en effet un facteur déterminant du non-usage : "Je me rappelle quand j'ai acheté le téléphone mobile, (...), je n'ai jamais pu lire les messages, je ne savais pas comment faire. J'ai été écœurée, je l'ai mis dans un coin et je ne m'en suis jamais servie. J'ai trouvé ça très compliqué ! (Germaine).

Outre le portable, certaines personnes nous ont raconté leurs expériences antérieures négatives avec d'autres TIC. La complexité de la technologie est toujours mise en avant. Pierre (72 ans), par exemple, nous a parlé de son expérience avec le magnétoscope. Il lui a fallu, en effet, deux mois pour comprendre le fonctionnement de cet outil qu'il lui a été offert par son fils. Pierre refuse ainsi d'avoir un lecteur DVD : "Maintenant que j'ai compris comment utiliser le magnétoscope, je n'ai pas envie de passer encore des mois à appendre comment faire fonctionner le DVD ». Pierre est aussi convaincu qu' avec Internet «ça va être pire ». Il avoue donc ne pas vouloir essayer cette technologie qu'il ignore et perçoit comme quelque chose de très complexe.

Enfin, l'expérience de certains réfractaires avec une autre technologie s'est avérée décisive dans le refus d'Internet : il s'agit de l'ordinateur.

\section{Le rapport à l'ordinateur :}

L'école de la Gestalt, en psychologie, considère qu'il n'existe pas de perception isolée ; celleci est au contraire initialement structurée (Kurt, 1935). Les processus de catégorisation et d'inférence, décelés par cette école, nous permettent d'examiner comment le non-usager, dans son schéma de perception d'Internet, peut catégoriser celui-ci en tant qu'objet informatique. Il lui est, en effet, possible de développer différents processus d'inférence dont la nature dépend de ses expériences avec d'autres outils de la même catégorie, comme l'ordinateur.

Puisque Internet est lié à l'ordinateur, ceux qui sont habitués de cette technologie peuvent se sentir plus disposés à utiliser le réseau que les personnes non familiarisées au monde de l'informatique. Nous avons ainsi questionné nos interlocuteurs sur leur rapport avec cet outil. Les réponses étaient hétérogènes mais nous ont toutes confirmé le fait que les perceptions et les expériences négatives de nos interlocuteurs avec l'ordinateur, ou encore l'absence complète d'expérience avec cet outil déterminent fortement le refus de l'usage d'Internet.

Parmi les sujets rencontrés, certains utilisent l'ordinateur ou ont eu l'occasion de l'utiliser, par obligation, notamment dans le cadre de leur travail. Ces personnes se sont montrées très critiques envers l'ordinateur. En effet, cet outil est souvent décrit par nos interlocuteurs comme une technologie « exaspérante », « assez compliquée » et «très fragile ». Nathalie, par exemple, utilise l'ordinateur dans son travail, mais elle ne l'utilise pas à la maison bien qu'elle en soit équipée. Elle avoue être très sceptique face à cette technologie et préfère ainsi garder, en parallèle, un système manuel pour gérer la bibliothèque où elle travaille. 
La complexité perçue est, selon Rogers, un facteur déterminant de la non-adoption d'une innovation. S. Ram -chercheur en sociologie de la consommation- postule également que le risque fonctionnel perçu d'une technique affecte le non-usage. Le risque fonctionnel peut être lié aux performances incertaines de la technologie (fragilité, risque de pannes...) et à la complexité de celle-ci. La complexité peut avoir selon Ram deux dimensions : «complexité de l'idée (est-elle facile à comprendre ?) et complexité de l'exécution (est-elle facile à mettre en application ?) »(Ram 1987, p. 210).

Les risques fonctionnels de l'ordinateur (pannes, virus ...) sont souvent évoqués par nos interlocuteurs et semblent déterminant du non-usage. Ils sont perçus suite à des expériences directes avec cet outil mais aussi suite à des expériences indirectes, comme le montre le discours de Germaine: "Je vois mon amie elle était contente quand elle l'a acheté, mais souvent quand je lui rends visite, elle me dit : "il faut que je téléphone à mon neveu car j'ai un problème avec l'ordinateur". Je trouve ça exaspérant! Et l'autre jour, je suis allée chez le médecin, j'arrive dans son bureau, il avait son ordinateur en panne. C'est pénible à la fin! Ces technologies sont une source de problèmes ».

Outre les risques fonctionnels liés à l'ordinateur, certains de nos interlocuteurs critiquent le support lui-même et ses composantes, notamment l'écran et le clavier :

«L'ordinateur me fait perdre mon temps. Moi j'ai une formation de base, c'est ABCD l'alphabet dans l'ordre. Parfois on tombe sur un clavier Quarty ou Azerty, donc on perd beaucoup de temps à chercher les lettres » (Jean-Éric).

«Je ne maîtrise pas l'ordinateur. Le fait de taper sur un clavier et de regarder l'écran, ce n'est pas évident! Il n'est même pas en ordre alphabétique, il faut trouver les lettres. Je ne me sens pas à l'aise devant cet appareil » (Michel, 33 ans).

Les réfractaires évoquent souvent l'inconfort de l'ordinateur et la difficulté pour eux de travailler sur un écran et de s'adapter au clavier compte tenu de l'absence de l'ordre alphabétique. Cela a certainement une influence sur le rejet de l'Internet puisque celui-ci est lié à l'ordinateur. Mais on ne peut pas faire porter aux ergonomes le poids du problème. C'est la volonté, le fait de vouloir utiliser l'outil en question, qui importe. Or, nos interlocuteurs n'affichent aucun intérêt envers Internet et l'outil informatique.

Plusieurs personnes rencontrées nous ont avoué avoir "un problème avec l'ordinateur». Florence (37 ans) nous a, par exemple, dit d'emblée qu'elle n'est pas tentée par Internet parce qu'elle ne supporte pas l'ordinateur : "J'ai du mal à supporter cette position à tenir la nuque penchée devant l'écran. Je n'ai jamais pu m'y mettre à l'ordinateur ».

Sylvie (34 ans) aussi nous a expliqué que l'une des raisons pour lesquelles elle ne veut pas utiliser Internet, est le fait que celui-ci soit lié à l'ordinateur : "Moi et l'informatique, ça fait deux (...), le simple fait qu'il y a un ordinateur dans l'histoire, ça ne me branche pas du tout! ».

Parmi les enquêtés, certaines personnes âgées nous ont parlé de l'occasion qu'elles ont eue d'approcher l'ordinateur avant de partir à la retraite. Elles nous ont avoué qu'elles ne sont pas arrivées à se familiariser avec cet outil. La complexité perçue de l'ordinateur et le manque d'habitude de manier ce genre de technologie sont les raisons avancées. L'ordinateur était, en effet, perçu comme un bouleversement total de leur manière de travailler. Ceux qui sont arrivés à «s'y mettre », par obligation, disent ne pas être prêts à le réutiliser. Ces retraités, et d'autres réfractaires interrogés, considèrent l'ordinateur comme un outil de travail qui doit être utilisé au bureau ; ils rejettent ainsi cet objet de l'univers domestique. Les propos suivants sont révélateurs de cette perception : "Qu'est-ce que vous voulez que je fasse avec un ordinateur, c'est un outil de travail. Moi je n'en ai pas besoin dans mon boulot et puis, pour 
ma vie personnelle, je ne vois pas à quoi ça peut me servir et c'est pareil pour Internet » (Jacqueline, 48 ans).

On a remarqué aussi chez quelques personnes rencontrées une confusion totale entre l'ordinateur et Internet. Ces objets sont considérés comme synonymes. En outre, certains réfractaires ne cachent pas leur méconnaissance de l'ordinateur et d'autres expriment leur désintérêt total envers cette technologie. Les réticences de ces non-usagers vis-à-vis de l'ordinateur s'expliquent en effet par un manque de formation; il est fréquent d'entendre dire : «Je ne sais pas comment ça marche et je ne cherche pas à comprendre » ou encore : "Je n'ai jamais eu l'occasion de me servir d'un ordinateur et en plus ça ne m'intéresse pas du tout!». Le manque de connaissance et de formation produit, comme l'a souligné Rogers (2003), des sentiments d'incertitude face à l'objet technique et provoque des résistances.

La méconnaissance de l'ordinateur ainsi que le manque d'intérêt envers cet outil constituent des freins majeurs à l'usage d'Internet. De même, l'expérience négative avec l'ordinateur influe sur le refus du réseau et l'absence totale d'expérience inhibe le processus d'usage (l'adoption, l'utilisation et l'appropriation).

\section{Le rapport des réfractaires aux médias « traditionnels »}

Nos interlocuteurs établissent souvent des comparaisons entre Internet et les médias dits «traditionnels ». Certains ont une vision très pessimiste et pensent que cette technologie va entraîner la disparition de tous les médias : "Internet ça va faire disparaître les livres, le cinéma, l'art et tout le reste»(Etienne, 35 ans). D'autres ont une vision plus optimiste : «Internet ne remplacera pas le livre et ne pourra pas non plus remplacer l'art, le théâtre, la peinture. Ce sont des valeurs sûres qui ne pourront jamais être remplacées par Internet» (Sylvie).

Plusieurs enquêtés insistent sur l'importance de la diversité des sources d'information. Il ne serait pas acceptable, en effet, qu'il devienne l'unique moyen d'information et de communication. D'où les déclarations suivantes : "Il ne faut pas qu'Internet soit pris sur le temps d'autres choses et que ça remplace les autres médias. La radio, la télé, le livre, tout ça est important» (Germaine); "Internet est partout, il parait qu'on peut tout faire avec. J'espère qu'il ne deviendra pas l'ultime média » (Daniel).

Préserver la diversité des moyens d'information et refuser le monopole d'Internet semblent des préoccupations importantes chez nos interlocuteurs. Ces derniers se plaignent de la «prédominance »du réseau et de son «emprise »sur les autres médias. Aujourd'hui, tout converge vers Internet, il est possible de lire, téléphoner, regarder la télé, écouter la radio en utilisant uniquement cette technologie. Ce scénario est perçu par nos interlocuteurs comme «très grave », « catastrophique ». Ils craignent qu'avec la prédominance d'Internet on risque de n'avoir qu'une seule vision, une seule culture, un seul discours. C'est pour cette raison que les réfractaires trouvent important de préserver la pluralité des sources d'information. Dans ce contexte, Ferid (41 ans) dit à propos d'Internet : "On appelle ça autoroute de l'information, c'est une autoroute, oui, mais le problème c'est qu'avec cette autoroute, on est en train de supprimer les nationales voilà ! C'est exactement ça!».

La cohabitation entre les médias et la variété des sources seraient nécessaires. La diversité de l'offre, dit André Vitalis, «permet l'expression de la liberté de choix du consommateur» (1994, p.41). Cette diversité doit concerner les supports mais aussi les contenus : le pluralisme 
de l'information. Dans cette «hypersphère » où Internet prend de plus en plus d'importance, il serait indispensable pour la réflexion, la culture et la liberté, de conserver une certaine place aux autres médias. En outre, ce qui semble plus important pour nos interlocuteurs c'est la qualité de l'information, et non la profusion des TIC.

Certains trouvent que les nouvelles technologies, symbolisées par Internet, "n'apportent rien » par rapport aux autres moyens existants : "Pour avoir des informations, la télé le fait très bien, les journaux le font très bien, la radio le fait très bien! Pourquoi en rajouter?", nous dit François (42 ans) à ce propos. Nos interlocuteurs insistent sur l'importance de la présence des autres médias et trouvent qu'Internet n'est pas «un outil indispensable » puisqu'il existe différentes alternatives en matière d'information et de communication.

Par ailleurs, certains ne nient pas les avantages que présente Internet notamment en termes de rapidité. Mais ils avouent être attachés à d'autres moyens de communication comme par exemple, le téléphone ou encore les lettres qu'ils trouvent «plus concrets», «plus personnels ». Pour eux, le téléphone ou les échanges épistolaires permettraient d'avoir une meilleure communication; de plus ils pensent, qu'avec ces moyens, le contenu du message serait transmis de façon plus sûre.

Enfin, les comparaisons les plus courantes qui ressortent des différents discours recueillis sont faites avec le livre, la radio et la télévision.

\section{Le rapport au livre et comparaison avec Internet :}

Les enquêtés font souvent des comparaisons entre Internet et le livre. Ces comparaisons sont quelquefois fondées sur une certaine connaissance du réseau et d'autres sur une méconnaissance de cette technologie.

Le refus d'Internet s'explique chez certains individus par un attachement à la «culture livresque » et au livre. On entend alors des expressions telles que : "Je suis complètement lecture »; "Internet ce n'est pas pour moi, je me satisfais encore du livre »; "Je préfère mon monde plein de papiers, mes livres, mon stylo et mon carnet $\gg$.

Plusieurs non-usagers considèrent Internet comme un danger pour le livre. Le risque culturel perçu est l'un des éléments déterminant de leur refus du réseau : "Le livre est menacé, les gens lisent de moins en moins, il n'y a plus que les "dinosaures" qui savent lire et apprécient la lecture », dit Jean-Éric. Internet serait perçu comme un bouleversement total : "l'ancien monde, celui de la lecture et de l'écriture, est en train de s'écrouler», nous dit Daniel. La culture livresque, la lecture, le livre «se meurent». «Nul besoin d'Internet pour lire. On a besoin d'Internet, en revanche, pour noyer le livre ». Cette citation d'Alain Finkielkraut (2001, p. 31) correspond à la pensée de plusieurs réfractaires interrogés.

Par ailleurs, pour certains de nos interlocuteurs, il n'y a pas que le livre qui serait menacé mais également les métiers qui lui sont rattachés. Certains sont persuadés que les librairies rencontrent aujourd'hui un problème de survie et qu'en conséquence le métier de libraire est menacé. Nathalie trouve également qu'Internet « représente un risque pour les métiers du livre », tel que celui du bibliothécaire. En effet, avec la généralisation d'Internet on a beaucoup parlé de l'avènement d'une grande bibliothèque virtuelle, d'une source d'information accessible à tout le monde. Plusieurs bibliothécaires, alors, nous explique Nathalie qui travaille dans ce domaine, ont vécu les débuts d'Internet comme une menace. Nathalie dit ainsi avoir « un problème personnel avec Internet »; et même si les bibliothèques existent encore, elle pense que celles-ci sont de moins en moins fréquentées. Nathalie 
compare les bibliothécaires et les documentalistes, notamment ceux qui sont réfractaires à Internet, aux copistes qui ont disparu progressivement avec la généralisation de l'imprimerie. Germaine trouve également qu'Internet représente une menace pour les professions du livre. Pour elle, cette technologie ainsi que la numérisation de l'information causeront d'ici quelques années la disparition des métiers liés à l'édition et à la vente du livre.

Malgré ce pessimisme et cette inquiétude concernant l'avenir du livre, considéré comme menacé face à l'évolution d'Internet, certains enquêtés ont une vision plus optimiste et sont certains que rien ne remplacera ce média. Celui-ci existe depuis des siècles et "restera éternel ». D'un point de vue culturel et éducationnel le livre serait une "valeur sûre», incomparable à Internet. Ce dernier ne pourrait pas être un outil d'appropriation de connaissances. Pour nos interlocuteurs il serait impossible de dissocier l'éducation, la culture et le savoir du livre. Pour Marie, par exemple, l'humanité ne pourra jamais vivre sans livres. Elle dit : "Internet ne pourra pas nous donner ce que le livre nous a fourni depuis des siècles. Au contraire Internet marginalisera cette culture. Le livre est la base de l'accès au savoir et à la culture, (...). Les hommes sans livres seront des abrutis ».

En effet, le livre serait perçu par nos interlocuteurs comme le symbole du monde du raisonnement et de la réflexion. Il nous apprendrait à penser et à développer notre sens critique. Les informations contenues dans les livres seraient considérées comme un apport de connaissance efficient, voire même comme ayant une capacité de rendre plus intelligent ; alors qu'Internet serait perçu comme un appauvrissement, un outil qui empêcherait la réflexion. Pour nos interlocuteurs, le livre serait donc rangé du côté de l'effort et Internet du côté de la passivité.

Internet est devenu, depuis quelques années, un nouveau support d'information utilisé par plusieurs personnes pour faire des recherches, pour lire des informations, des articles, voire des livres en texte intégral. La lecture sur Internet qui exige le passage par un écran est rejetée et critiquée par nos interlocuteurs. Cette pratique présenterait des inconvénients multiples. En effet, «[celle-ci] n'est pas considérée comme efficace. (...) le numérique décourage une lecture savante en privilégiant la vitesse, l'éclatement, la superficialité plutôt que la réflexion, l'approfondissement, la critique. (...). La lecture sur papier est considérée comme la seule légitime et confortable »(Salaün, In Guichard, 2001, p.114). La lecture sur ordinateur présenterait des problèmes qui seraient liés à la nature des écrans et à la qualité des postes de travail. On parle depuis quelques années des enjeux médicaux de cette nouvelle pratique de lecture : conséquences d'ordre physique dues aux effets de la position assise et à la fatigue des yeux. Bien que des efforts aient été faits dans ce domaine, nos interlocuteurs trouvent que la résolution des écrans demeure insuffisante pour une lecture commode et confortable.

Parmi les autres incommodités, nos interlocuteurs citent celles liées :

- au temps de la lecture qui serait nettement plus long que la lecture sur papier ;

- au problème de la posture dans la mesure où, avec l'ordinateur, il ne serait pas pratique de lire partout où l'on veut. En effet, le livre rendrait plus autonome, il serait facilement transportable et n'aurait pas d'impératif d'unité de lieu. Les propos suivants sont révélateurs de cette perception : "Le livre on peut le prendre n'importe où sans problème, sans coupure de courant, sans problème de virus » (Jean-Paul, 41 ans) ; «Sur un livre, on peut marquer des annotations, on peut lire plus attentivement, ce n'est pas comme à l'écran » (Florence).

Les non-usagers d'Internet considèrent que l'écran d'un ordinateur ne pourra jamais donner cette satisfaction que donne le livre. L'écran «fait perdre les émotions attachées à la réalité », il «dématérialise tout». Nos interlocuteurs trouvent également la lecture sur écran «bizarre », «lourde », «inconfortable », «fatigante » et « désagréable ». L'écran lui-même, 
est souvent qualifié de «froid», «artificiel» et «impersonnel». Le livre par contre est qualifié de «concret», «palpable». Il y aurait en effet un rapport affectif et tactile avec le livre, ce dernier solliciterait les sens.

Les non-usagers comparent aussi la souplesse du livre avec la rigidité de l'ordinateur. Ils trouvent plus «agréable» de feuilleter le livre, de «l'amener partout», que d'être «enchaîné » devant Internet. La lecture du livre aurait un aspect intimiste. Il y aurait une sorte de dialogue silencieux entre le lecteur et l'auteur qui conduirait, selon nos interlocuteurs, à une plus grande appropriation du contenu : «On a un rapport au bouquin qu'on n'a pas avec Internet. Je n'ai pas de liens avec les phrases que je vois défiler sur un écran », dit Germaine dans ce contexte.

Parmi les réfractaires rencontrés, certains ont eu l'occasion d'observer l'usage d'Internet. Ainsi, ce n'est plus seulement la lecture sur écran qui est refusée et comparée avec le livre mais également la lecture de l'hypertexte : "Sur Internet on doit toujours cliquer ou taper, c'est vraiment énervant! Le livre on le lit tranquillement, sans se prendre la tête » (Etienne); «On ne peut pas vraiment lire sur Internet, on doit toujours aller d'un site à un autre. On ne peut pas se concentrer. C'est soûlant! »(Michel).

La dynamique du Web peut transformer la lecture en une activité «agitée » où le lecteur peut rester à la surface du sens du texte. Il peut être emporté dans un système d'images et de fragments de textes qui peuvent être oubliés dès qu'ils ont été vus. En effet, la navigation au moyen de la souris et des cliques, provoque des déplacements extrêmement rapides qui ne sont pas très favorables à la lecture. Dans cet ordre d'idée, Alain Cordier dit : «Lire, c'est bien souvent adopter une prise en main lente et méditative. Les auteurs anglo-saxons ont un terme pour décrire cela : deep-reading, une lecture profonde $\gg(1999, \mathrm{p} .15)$. Or de ce point de vue, Internet ne permettrait pas, selon nos interlocuteurs, d'envisager une telle lecture.

Le livre semble, en définitive, l'un des médias préféré de plusieurs réfractaires interrogés. Il est mis en valeur par la majorité de nos interlocuteurs qui vouent ainsi aux gémonies l'Internet.

\section{Le rapport à la radio et comparaison avec Internet :}

La radio fait partie également des médias les plus utilisés chez plusieurs enquêtés. Cela est reflété par des expressions telles que: «Je suis plus radio moi»; «J'écoute beaucoup la radio », «Elle est tout le temps branchée ».

Les discours recueillis mettent en exergue la radio par rapport à Internet. Pour nos interlocuteurs, la radio permettrait une plus grande liberté de mouvement qu' Internet dont l'emploi obligerait l'utilisateur à rester devant l'écran de l'ordinateur. Cette liberté s'apparente à la possibilité d'effectuer d'autres activités au moment de l'écoute. Il s'agit le plus souvent de travaux domestiques et notamment des tâches ménagères. Les enquêtés disent préférer la radio car « elle permet de garder une certaine autonomie ».

Par rapport à Internet, la radio serait également perçue comme plus facile d'accès, «elle est transportable partout » et «moins chère » que l'ordinateur et la connexion. Cet argument est avancé par certains qui critiquent le fait que la radio soit aujourd'hui accessible par Internet. Pour Michel, par exemple, la radio serait un outil plus simple à utiliser; il trouve « débile » de se connecter à Internet pour l'écouter : "Je préfère acheter une petite radio à 5 euros que je peux prendre partout avec moi que d'acheter un ordinateur qui coûte quand même hyper cher et de prendre un abonnement que je dois payer chaque mois ». 
De façon générale, la radio n'est pas critiquée par nos interlocuteurs, sauf pour ce qui est des stations considérées par certains comme «commerciales ». Ils disent ainsi être agacés par les publicités et la «mauvaise musique » passées par ce genre de radio.

Enfin, certains enquêtés avouent avoir perdu l'habitude d'écouter ce média à cause notamment de leur pratique télévisuelle.

\section{Le rapport à la télévision et comparaison avec Internet :}

Certains enquêtés se considèrent comme de "gros consommateurs" de télé. Toutefois, ce média est critiqué par un bon nombre de nos interlocuteurs qui font un rapprochement entre la télévision et Internet. Pour eux, ces deux technologies entraîneraient une passivité d'esprit chez leurs utilisateurs.

Ceux qui préfèrent la télé considèrent cet outil comme un média qui permettrait de rassembler les membres de la famille et d'avoir des sujets de discussion. Elle serait donc avantagée par rapport à Internet qui serait considéré comme un "média" individualiste. Dans ce contexte, Dominique Wolton note que la télévision est la seule capable de créer du lien social dans cette « société individualiste de masse ». Elle sert «à rassembler des individus et des publics que tout sépare par ailleurs et à leur offrir la possibilité de participer individuellement à une activité collective. C'est l'alliance bien particulière entre l'individu et la communauté qui fait de cette technique une activité constitutive de la société contemporaine » (Wolton, op.cit., p.73). Certains enquêtés plaident pour la télévision contre l'Internet qui accentuerait, selon eux, l'individualisme et l'isolement. La télévision fait partie de la vie quotidienne de la majorité des français. En outre, en tant que moyen de communication, elle aurait un rôle inestimable et constituerait un des liens sociaux les plus forts. La télévision, "incarne la culture de masse » alors qu' Internet est considéré par certains de nos interlocuteurs comme « un outil élitiste» : «La télévision est accessible à tous. Tout le monde peut s'en servir alors qu'Internet il faut savoir l'utiliser, il faut un minimum de connaissances », dit Céline (37 ans).

Parmi les autres arguments avancés par les enquêtés qui défendent la télé par rapport à Internet, on trouve ceux liés au confort et à la simplicité d'utilisation de ce média : "il est plus confortable de regarder la télé, je peux être allongé sur mon canapé, ou couché dans mon lit, c'est agréable!"; "c'est simple de mettre en marche la télé, par contre aller sur Internet demande un savoir-faire ». L'ordinateur et Internet demanderaient la présence continue et active de l'usager pour fonctionner, alors que la télévision nécessiterait seulement d'être allumée. La pratique télévisuelle n'empêcherait pas non plus de faire simultanément d'autres activités : "La télé on peut l'allumer et faire d'autres choses, on peut tricoter, manger, faire le ménage et avoir un oil sur la télé. On n'est pas obligé d'avoir les yeux scotchés sur l'écran et les mains sur le clavier» (Michel).

En outre, les interlocuteurs qui disent préférer la télévision, n'admettent pas le fait qu'Internet puisse servir à regarder la télé. Pour eux il serait inadmissible de regarder leurs émissions sur l'ordinateur qu'ils considèrent comme un outil de travail.

Le succès de la télévision est exceptionnel depuis des décennies. Tout le monde, ou presque, la regarde et elle continue ainsi à faire « l'ordre du jour » et à susciter des discussions. Mais plusieurs personnes la critiquent aussi et s'en méfient. Parmi nos interlocuteurs, certains avouent être réfractaires à la télévision autant qu'à Internet. Jean-Paul trouve par exemple la télévision d'aujourd'hui «un peu surréaliste exactement comme Internet ». Pour Daniel, 
également, ces deux "médias" sont semblables : «pour moi ce sont deux outils dangereux, c'est virtuel, les gens vivent par procuration au travers des écrans ».

Internet et télévision sont alors dénoncés et accusés des mêmes torts. Ils seraient considérés comme dangereux pour les enfants et les adolescents. Ils auraient les mêmes conséquences négatives et les mêmes risques, notamment pour la culture livresque dans la mesure où «les gens préfèrent regarder la télé, se connecter à Internet plutôt que lire », nous dit Daniel avec regret.

Certains enquêtés reprochent à Internet sa similitude de fonctionnement avec la télévision. Pour eux, ces deux médias seraient entachés par les publicités et leurs pratiques encourageraient la routine et la passivité. En outre, d'autres sont convaincus que les sites sur Internet sont, comme les programmes télévisuels, «très marqués par une dominance américaine » : télé réalité, séries, films américains, etc. Certains de nos interlocuteurs parlent d'ailleurs d'une «américanisation du monde » engendrée par la télévision et accentuée avec le développement d'Internet. Les propos suivants sont révélateurs de cette perception : «Internet est exactement comme la télé, un outil qui incarne la culture et les valeurs américaines » (Richard); "La télévision d'aujourd'hui est complètement américanisée. Elle est envahie par les émissions et les séries américaines. Avec Internet c'est encore pire! C'est un outil inventé par les Américains pour une américanisation des esprits »(Jean-Paul).

Ces réfractaires attribuent à la télévision et à Internet un pouvoir d'uniformisation et de corruption des modes de vie et des visions du monde. Le refus de l'Internet et de la télévision «leur permet d'échapper aux déterminismes de ces technologies ». Ils considèrent ainsi que leurs connaissances et leur culture, n'étant pas entachées par ces outils, sont «pures » et «exactes ». Ces dispositifs seraient considérés en effet comme des instruments de contrôle social, des systèmes régis par la logique économique et politique. Le non-usage de ces outils représenterait pour eux un signe distinctif d'autonomie. Il leur permettrait d'échapper à l'idée que leurs pensées et leurs actions soient dictées par ces médias.

Par ailleurs, le fait que ces deux technologies soient dotées d'un écran est une autre similitude évoquée et "détestée" par certains réfractaires : "Je ne regarde pas la télé, je n'aime pas avoir les yeux scotchés sur un écran! Et Internet c'est comme la télé ça marche avec un écran » (Marie) ; "L'écran de télé est nuisible pour les yeux, pourtant il est généralement placé à deux/trois mètres. Que dire alors d'un écran d'ordinateur qui est placé plus près que ça. (...) En fait l'écran m'étourdit » (Daniel).

Télévision et Internet semblent en définitive des technologies similaires dans les représentations de certains enquêtés. Parmi ceux qui sont convaincus de cette similitude, certains ne disposent pas de télé et affirment comme le reste de nos interlocuteurs leur nonusage volontaire d'Internet.

Aujourd'hui, il n'est pas fréquent de prendre une position critique ou hostile vis-à-vis des technologies de l'information et de la communication dans la mesure où le flot d'éloges les définit d'entrée comme des dispositifs bienfaisants et prodigieux. Pourtant, notre enquête nous a montré que certains individus ont une vision moins optimiste d'Internet et de certaines TIC. 


\section{Conclusion}

Les caractéristiques perçues des TIC s'avèrent des facteurs déterminants dans le rapport des enquêtés à ces dispositifs. Les discours recueillis montrent que les TIC (Internet, l'ordinateur, le téléphone portable, les appareils numériques, ...) sont perçues comme incompatibles avec le mode de vie de nos interlocuteurs. Les individus sont généralement amenés à s'interroger sur la compatibilité des innovations avec un ensemble d'éléments auxquels ils sont attachés. En effet, ils fondent en partie leur évaluation d'une technologie donnée sur la compatibilité perçue de celle-ci avec leur mode de vie, leurs habitudes et préférences. Plus une innovation est conforme à ceux-ci et moins importante devrait être la résistance à son égard.

Les préférences (notamment en matière d'information et de communication), les pratiques techniques et médiatiques habituelles des réfractaires à Internet semblent en définitive des facteurs influents sur leur refus du réseau.

La complexité et les risques perçus de la technique sont aussi des éléments qui ont été mis en avant dans les propos de nos interlocuteurs : risque fonctionnel (fragilité, obsolescence, incertitude de performance); risque social (détérioration du lien social, individualisme), risque culturel (menace pour la culture livresque, risque de l'emprise d'Internet sur les autres médias), risques physique et psychologique (crainte d'être aliéné et appréhension d'être dominé par la technologie, conséquences d'ordre physique des incommodités perçues). Ces risques décelés déterminent les faibles possibilités d'essai et de transfert technique ainsi que le non-usage.

Par ailleurs, nous pouvons également attester que certaines caractéristiques propres aux enquêtés déterminent fortement leur rapport aux TIC et à Internet.

En effet, les attitudes, les représentations et les expériences (directes ou indirectes) qu'ils ont des nouvelles technologies, le non-besoin, le désintérêt envers ces outils, l'attachement à certains dispositifs et le refus d'autres, les caractéristiques sociodémographiques dont notamment l'âge de certains enquêtés, s'avèrent des éléments en corrélation avec le non-usage d'Internet et le rapport aux innovations chez nos interlocuteurs.

Notre article intitulé «Comprendre le non-usage technique : réflexions théoriques » a permis de souligner la relation entre le non-usage et les caractéristiques de la technique ainsi que celles de l'individu. La présente recherche, basée sur une enquête de terrain, nous a permis de confirmer l'influence de ces caractéristiques sur le non-usage technique. Cependant, cette étude n'a pas pu mettre en évidence l'influence de la caractéristique «efficacité de soi » sur le non-usage. Les réfractaires interrogés n'ont pas évoqué un sentiment de manque de confiance en soi face aux nouvelles technologies.

Par ailleurs, certains d'entre eux ont exprimé une forme de «dogmatisme ». Des interviewés rencontrés perçoivent en effet les nouvelles technologies comme «une source de bouleversement ». Ils associent les innovations techniques à de nouvelles pratiques qui exigent des changements et des ajustements inacceptables.

Pour le professeur de psychologie sociale M. Rokeach -dont les travaux sont consacrés à l'étude des systèmes de croyances et d'attitudes- les individus qui se sentent menacés par la perspective du changement sont « dogmatiques ». Ils seront donc plus résistants à la nouveauté.

Selon Rokeach (1960), le dogmatisme est caractérisé par l'esprit fermé de certaines personnes. Cet esprit renvoie à un système de croyance clos, il reflète une intolérance à 
l'égard de toute nouveauté. Il serait hermétique au changement et ancré dans la tradition. L'on peut dire donc qu'un individu « dogmatique », attaché à ses habitudes et qui voit dans l'utilisation d'une nouvelle technologie une menace risquant de les perturber, va très probablement refuser ce dispositif. Dans ce contexte, S. Ram note que «plus le consommateur fait preuve de dogmatisme plus la résistance à l'innovation est forte » (1987, p.210). Le dogmatisme apparaît donc comme une forme de résistance au changement qui permet de justifier le non-usage des innovations chez certains individus.

Il est par ailleurs important de souligner le fait que nous ne pouvons considérer l'ensemble de nos interlocuteurs comme dogmatiques. Le discours tenu par des interviewés ne permet pas de les situer comme tels. Il s'agit notamment des propos qui renvoient à leur détermination à échapper au contrôle social des TIC qu'ils considèrent comme des systèmes régis par les logiques économiques et politiques.

Le discours de certains interrogés, qui ont exprimé un fort attachement à des pratiques habituelles différentes de celles exigées par les nouvelles technologies, reflète une forme de dogmatisme.

Ce dogmatisme, qui réfère à une résistance aux changements techniques, affecte leur rapport aux TIC. Toutefois, il n'est pas judicieux d'expliquer le refus de l'Internet et le rapport aux autres TIC par cette caractéristique. Le non-usage de nos interlocuteurs est volontaire et leur rapport aux TIC est fondé sur un choix délibéré.

La technique n'est pas neutre. Les enjeux et les caractéristiques perçus des TIC déterminent fortement le non-usage de nos interlocuteurs et leur rapport à la technique.

Dans cet article nous avons essayé de présenter une réflexion empirique sur la question du non-usage et plus spécifiquement sur le refus de l'Internet et le rapport des réfractaires aux autres TIC et aux médias dits traditionnels.

D'après une étude de l'Insee, publiée en mars 2011, un quart des Français déclarent n'avoir jamais utilisé Internet. Parmi les raisons citées : le coût de l'ordinateur ou de l'abonnement, le manque de compétences et enfin la non-utilité (4). Par rapport aux usagers d'Internet, les nonusagers délibérés, ne sont pas nombreux, mais ils existent et il semble important de s'intéresser davantage aux raisons de leur non-usage.

La problématique du non-usage technique ouvre de nombreuses perspectives de recherche. En sciences de l'information et de la communication, les études abondantes sur les TIC semblent focalisées sur la question des usages sociaux et professionnels de ces outils, négligeant souvent la problématique du non-usage. Avec le développement accéléré des technologies on ne peut plus passer outre les formes de résistance.

On étudie souvent les TIC à travers leurs usages et usagers. Il serait intéressant d'étudier ces objets techniques par le biais des non-usagers. Les représentations de ces derniers peuvent participer à la définition sociale d'une TIC, au même titre que celles de ses utilisateurs. 


\section{Références Bibliographiques}

Balloffet, Pierre, Boulaire, Christèle (1999), « Freins et motivations à l'utilisation d'Internet : une exploration par le biais de métaphores », Recherche et applications en Marketing, $\mathrm{n}^{\circ} 14$, p.28-39

Bardin, Laurence (1977), L'analyse de contenu, Paris : PUF

Blanchet, Alain, Gotman, Anne (1992), L'Enquête et ses méthodes : L'entretien, Paris : Ed. Nathan

Boudokhane Lima, Feirouz (2006), «Comprendre le non-usage technique: réflexions théoriques », Les enjeux de l'information et de la communication

Article en ligne : http://w3.u-grenoble3.fr/les_enjeux/2006/Boudokhane/index.php

Breton, Philippe (2000), Le culte de l'Internet: Une menace pour le lien social ? Paris : La Découverte

Cordier, Alain (1999), «Le livre numérique : Internet et la pensée », Communication et langages, $\mathrm{n}^{\circ} 122$, p.11-18

Djoudi, Mahieddine (2001), «Conception d'assistants à la navigation sur l'Internet » (p.165173), in Guichard, Eric (dir.), Comprendre les usages de l'Internet, Paris : Ed. Rue d'Ulm/Presses de l'Ecole normale supérieure

Finkielkraut, Alain (2001), «Fatale liberté », Internet l'inquiétante extase, Paris : Mille et une nuits, Fondation du 2 mars

Gras, Alain (2003), Fragilité de la puissance : se libérer de l'emprise technologique, Paris : Fayard

Koffka, Kurt (1935), Principles of Gestalt psychology, London : Routledge \& Kegan Paul

Mallein, Philippe, Privat, Gilles (dir.) (2003), Ces objets qui communiquent, Paris, Lavoisier, Numéro spécial des cahiers du numérique, vol. $3, n^{\circ} 4$

Moliner, Pascal, Rateau, Patrick, Cohen-Scali, Valérie (2002), Les représentations sociales : pratique des études de terrain, Rennes : Presses universitaires de Rennes

Questions de communication (2010), «Repenser le non-usage des TIC », Dossier coordonné et présenté par Catherine Kellner, Luc Massou, Pierre Morelli, $N^{\circ} 18$

Ram, Sudha (1987), «A model of innovation resistance », Advances in consumer research, $n^{\circ}$ 14 , p.208-212

Chartier, Roger et al. (1997), Histoire de la lecture dans le monde occidental, Paris : Seuil

Rogers, Everett (2003), Diffusion of innovations, New York : Free Press 
Rokeach, Milton (1972), Beliefs, attitudes and values : A theory of organization and change, San Francisco : CA, Jossey-Bass

Rokeach, Milton (1960), The open and closed mind : investigations into the nature of belief systems and personality systems, New York: Basic Books

Salaün, Jean-Michel (2001), « Documents numériques et universités françaises » (p.112-117), in Guichard, Eric. (dir.), Comprendre les usages de l'Internet, Paris: Ed. Rue d'Ulm/Presses de l'Ecole normale supérieure

Vitalis, André (1994), «La part de citoyenneté dans les usages »(p.35-43), In Vitalis, André (dir.), Médias et nouvelles technologies : Pour une socio-politique des usages, Rennes : Ed. Apogée

Wolton, Dominique (2000), Internet et après ? Une théorie critique des nouveaux médias, Paris : Flammarion

\section{Notes}

(1) Les propos des réfractaires à Internet sont mis en italique pour les différencier des citations des auteurs.

(2) Voir par exemple le rapport d'information de la mission d'information commune sur les droits de l'individu dans la révolution numérique, enregistré à la Présidence de l'Assemblée nationale le 22 juin 2011 et intitulé : Révolution numérique et droits de l'individu : pour un citoyen libre et informé. URL: http://www.numerama.com/media/Rapport\%20V1Revolution-Numerique.pdf

(3) Voir à ce sujet :

- Marquié, J.-C., Baracat, B. (2001), «Les plus de 45 ans dans un contexte technologique mouvant » (pp. 359-375), in J.-C. Marquié et al. (Eds.), Le travail au fil de l'âge, Toulouse : Editions Octarès

- Rouet J.-F., Cent fenêtres sur Internet: Une étude des représentations et des usages des technologies d'information et de communication dans le grand public, avril 2003

[En ligne] http://www.mshs.univ-poitiers.fr/100fenetres

(4) Voir article en ligne, Reuters France : « «La fracture numérique se réduit peu à peu en France ». URL : http://fr.reuters.com/article/technologyNews/idFRPAE72100O20110302 